\title{
Acknowledgement to Reviewers of Challenges in 2019
}

\author{
Challenges Editorial Office \\ MDPI, St. Alban-Anlage 66, 4052 Basel, Switzerland
}

Published: 22 January 2020

The editorial team greatly appreciates the reviewers who have dedicated their considerable time and expertise to the journal's rigorous editorial process over the past 12 months, regardless of whether the papers are finally published or not. In 2019, a total of 31 papers were published in the journal, with a median time to first decision of 24 days and a median time from submission to publication of 42 days. The editors would like to express their sincere gratitude to the following reviewers for their generous contribution in 2019:

\begin{tabular}{ll} 
Ahmadvand, Seyedsaeid & Islam, Mohammad Mazharul \\
Ameta, Sandeep & Jankowska, Marta \\
An, Chunjiang & Karabasevic, Darjan \\
Assari, Shervin & Kelly, Daniel \\
Benatar, Solly & Keramydas, Christos \\
Berber, Nemanja & Kingsley, Jonathan \\
Blaise, Nguendo Yongsi & Kirakosyan, Lyusyena \\
Brack, Andre & Kjellen, Marianne \\
Busby, Joshua W. & Kondo, Michelle C. \\
Capraro, Valerio & Kurppa, Sirpa \\
Chen, Chong & L. Schmitz, Cathryne \\
Cole, Jennifer & Liao, Chen \\
Comas-Garcia, Andreu & López-Sánchez, Jesús \\
Craig, Jeffrey & Macioce, Fabio \\
Crnogorac-Jurcevic, Tatjana & Madra-Sawicka, Magdalena \\
D'Anna, Linda & Magliocco, Adriano \\
Davidove, Marian E. & Martin, Wanda \\
Dettori, Marco & McFarlane, Ro \\
Díez Ruiz, Fernando & Melo, Pedro \\
Dillon, Denise & Mizuuchi, Ryo \\
Domingo-Irigoyen, Silvia & Myers, Samuel \\
Drawert, Brian & Najjar, Mohammad \\
Elbarazi, Iffat & Nam, Taewoo \\
Fairen, Alberto & Nazarko, Lukasz \\
Featherstone, Mark & Occelli, Florent \\
Fernando, Julian & Okulicz-Kozaryn, Adam \\
Fojcik, Marcin & Ong, Corinne \\
Foucher, Frédéric & Papadakaki, Maria \\
Glombik, Konrad & Pateiro, Mirian \\
Gray, Tim & Petrikova, Ivica \\
Greblikaite, Jolita & Plichta, Marta \\
Gu, Fu & Pombo, Ana \\
Hall, James & Price, Dan \\
Hancock, Trevor & Ribeiro, Helena \\
Heo, Hyun Hee & Rosa, Flavio \\
& \\
\hline
\end{tabular}


Rugheimer, Sarah

Sacco, Pier

Samoila, Cornel

Shaw, Margie Hodges

Song, Zhaoli

Sybesma, Wilbert

Vanegas, Diana C.
Varma, Vijaykumar B.

Voss, Joachim

Warner, Elyse

Webb, Darren

White, Brandi M.

$\mathrm{Wu}$, Jianyong

(c) (i)

(C) 2020 by the author. Licensee MDPI, Basel, Switzerland. This article is an open access article distributed under the terms and conditions of the Creative Commons Attribution (CC BY) license (http://creativecommons.org/licenses/by/4.0/). 